\title{
Development Overview and Valuation Analysis of Bilibili
}

\author{
JiaLing Deng ${ }^{1, \dagger}$,Ying Yu Han ${ }^{2, \dagger}$, GuoXu Zhou ${ }^{3, \dagger}$ and Xuyang Zhou ${ }^{4, \dagger}$, \\ ${ }^{1}$ Jiangsu Tianyi High School, tydj1040513@163.com \\ ${ }^{2}$ Wuxi Foreign Language School, 2799586865@qq.com \\ ${ }^{3}$ Hailiang Foreign Language School, yangheqizhero@gmail.com \\ ${ }^{4}$ Ulink College of Shanghai, SimonZhou1210@gmail.com \\ These authors contributed equally
}

\begin{abstract}
Bilibili, as a novel type company different from the traditional video business, is a highly concentrated cultural community and video platform for the young generation in China, which attracts a large number of users and content creators. This paper investigates the development process and business value of Bilibili through analysis. Specifically, in order to analyze the position of Bilibili in the industry and the future development, we present the comparison and measures with industry competitors, the transformation process from initialization to diversification and the adjustment of the operation model and profit direction. Besides, the reasons for the final scale expansion are also discussed. Based on the valuation of Bilibili, the current price of Bilibili is consistent with the market expectation. Moreover, its business also has the potential growth capacity, which further shows that Bilibili is unique and scalable in the current industry. These results provide inspiration for the future development and in-depth exploration of the short video industry
\end{abstract}

Keywords: Bilibili, Development, Operation, Valuation

\section{INTRODUCTION}

With the popularity of the internet, the entertainment path of human beings has also changed, where many people chose to relax by watching videos online. On this basis, Bilibili, a video-sharing website, is becoming more and more popular among both young and middleaged people in China. It is called YouTube in China and is expected to attract more users in the future. Bilibili has generated a unique Danmaku culture which is pursuit by the generation $\mathrm{Z}+$ (the demographic cohort of individuals in China born from 1985 to 2009). They will become the main consumers in the near future, i.e., it is important and necessary to analyze the company's current situation and future expectations.

Bilibili New Year's Eve event has become the company's signboard, Liu and Wang conducted a study on the Bilibili New Year's Eve party as a kind of communication media and its transition to productization. Meanwhile, it also analyzes the logical relationship between media productization and circle propagation. This survey shows that for Bilibili, the use of media products (e.g., the New Year's Eve party) is not only a marketing method but also a unique communication way of social media. This will power the growth of Bilibili's user base [1]. Fu and Lin compared Bilibili's New Year's Eve party with other TV channels through horizontal comparison. The content setting and operation thinking of the Bilibili evening party are analyzed. It includes the spontaneous gathering caused by content, the group resonance caused by collective memory, the transition from single traffic to group traffic, from cross-boundary mixing to cultural integration. These features provide reference experience for relevant media practice and put forward suggestions on the construction of cyberspace culture for the future development of Bilibili's media form [2]. Gao explained the advantages of Bilibili in strategic transformation through the analysis of successful marketing factors of Bilibili's New Year's Eve party. According to the in-depth excavation and analysis of the problems exposed by the marketing of the Bilibili evening party, this paper provides the corresponding potential solutions and forecasts the future development of Bilibili [3]. 
Zhao et al use data of the investment channel and scale of Bilibili to study the modern corporate financial strategy. Starting with Bilibili's strategic environment, based on financing, investment, distribution, and social responsibility, this paper also tried to analyze Bilibili's advantages and disadvantages from its financial strategy. Finally, from the perspective of social responsibility, it is concluded that the relationship between corporate financial strategy and social responsibility is interactive. It is optimized that Bilibili should establish a financing structure with balanced risk of stakeholders and innovate financing methods as well as formulate a relatively stable dividend distribution plan to enhance capital confidence [4]. Yuan utilizes a series of statistics about Bilibili's cash flow structure for carrying out management, investment, and fundraising activities to analyze the structure and variation tendency of the cash flow. She calculated the cash coverage ratio and the ratio of cash to current debts to analyze the quality of Bilibili's cash flow. It is concluded that Bilibili's cash flow has poor quality, and operating activities do not generate net cash inflows. The business's operation is largely dependent on external fundraising [5]. Gao et al use the financial data to analyze the sustainable growth prospect of Bilibili company. In their studies, the capability, stability as well as the future growth are investigated. It is found that Bilibili should make full use of the advantage of having lots of two-dimension users to enhance the development potential. It should scale the current profit model to ensure sustainable development [6]. Jiang conducted the research mainly about the way of getting profit, factors of profit, and the financial analysis of Bilibili's operation. As two-dimensional space became the most popular domestic firm, BILI got investment from Tencent and Alibaba. This article uses the method called document analysis and research on the special dissertation to analyze four aspects, profit, operating, debt and development capacity. It also mentioned that the most profit comes from games, live streaming, advertisement etc. Besides, they list a few improvement ways as well. In general, the total revenue has grown for four years while the net profit is negative [7].

As for the operation fields, Zhu conducted the research mainly about how the special characteristics of Bilibili gain so much attraction from the young. The article mentioned that the most competitive aspect for BILI is the culture difference (compared with the other two apps). Some ways of getting profit are also concluded. It lists the problems BILI faced at that time which are consumers not stable, the quality of video reduced, etc. The main purpose is to solve these problems by using corresponding ways like upgrade the membership or strength the platform management. Because two-dimensional space is not that easy to accept, BILI needs to focus on building the connection with the market [8]. Weng and Dai conducted the research to discuss the different operation ways between LETV and Bilibili. The study shows that LETV had huge investment projects like technology and set lots of platforms that caused the deficit. Compared with LETV, BILI focused on attracting the fixed group and had a joint supply to exploit the advantages to the full. The comparison between users and marketing projects is also used [9]. Rong et al. has explored the determinants of user stickiness in the online video industry through platform theory. According to their research, the online video industry mainly comprises two patterns of the business model (the merchant platform and the twosided platform). A theoretical model has been built by them to explore the essential difference between the two, which enables them to jump to the conclusion ultimately that the network effect is stronger in the twosided platform than in the merchant platform. Besides, an indirect network makes customers more multihoming. They also pointed out that price is not a significant influencing factor in user stickiness [10]. Lin et al. have researched the motivations of internet users to share videos and spend more time on video-sharing websites. A model based on Uses and Gratification Theory and Social Cognitive Theory incorporating key determinants of website stickiness was built by the authors to develop their research. Their results indicated that continuance motivation and sharing behavior were important antecedents of YouTube stickiness and mediated the impacts of need, personal, and environmental factors [11]. Yang et al. has investigated consumer attitudes toward advertisement while they are watching online videos and the effects on shopping intention and purchase behavior. They used Brackett and Carr's (2001) Web Advertising Attitudes Model and combined it with the theory of reasoned action and the flow theory to develop their research. Entertainment, informativeness, irritation, and credibility were proved by them to have a shopping influence on purchase attitudes, while flow does affect shopping intention and purchase behavior [12].

This passage mainly analyzes Bilibili Inc.'s competitor by comparing it with other video platforms, e.g., iQIYI and Netflix. Subsequently, it focuses on some aspects, including New Year's Eve, the business's bottleneck and transformation. Then, Bilibili's operation model and profit are also analyzed, and rationable suggestion is then proposed to help the company earn more profit. Finally, the Discounted Cash Flow model and $\mathrm{P} / \mathrm{S}$ ratio are utilized to make a valuation of the company and predict its future growth. The data of the company's operation expenditure and market capitalization are adopted to calculate the $\mathrm{P} / \mathrm{E}$ ratio. Moreover, the free cash flow is predicted to evaluate the value. 


\section{COMPETITION IN THE INDUSTRY}

Except for Bilibili, some other video platforms are also commonly used in China, including iQIYI, Netflix, TikTok, Volcano, etc. As for iQIYI, in 2019, the total revenue was 29 billion yuan and the net deficit was 10.3 billion yuan. Since it was established, iQIYI has always been at a loss. According to the last 3 years' data, the amount of loss was 3.7 billion, 9.1 billion, and 10.3 billion yuan, respectively. The main reason for iQIYI's loss was its content costs, which was 30.3 billion in total. Especially in 2019, it spent 22.2 billion on content procurement and production expenses. In terms of Netflix in 2019, the total revenue is $\$ 201.56$ billion, the main business cost is $\$ 12.440$ billion, the marketing expenditure is $\$ 2.652$ billion, the research and development expenditure is $\$ 1.545$ billion, and the general and administrative expenses are $\$ 914$ million, i.e., the operating profit is $\$ 2$.6billion. The second part is the company's debt payments and income. Adding with negative 5.4 million dollars, the pre-tax profit is $\$ 2.06$ billion, the net profit after the tax payment is $\$ 1.867$ billion.

However, Bilibili shows about $50 \%$ of the raised funds will be used to improve the content, including acquiring and investing in related goods and services, producing premium paid content, providing infrastructure, and creating incentive mechanisms for creators. Besides, $20 \%$ will be devoted to the research and development of information technologies such as $\mathrm{AI}$, as well as the development of self-developed games to improve user experience and enhance commercialization capabilities. In addition, $20 \%$ will be spent on marketing and sales to drive user growth and brand stability. The remaining $10 \%$ is for general business purposes and working capital needs. From 2018 to 2020 , Bilibili's revenue increases from 4.13 to 12 billion yuan, while a net loss of 565 million yuan, 1.304 billion yuan, and 3.054 billion yuan, respectively. From 2015 to 2017, Bilibili's net loss were 569 million yuan, 1.185 billion yuan, and 571 million yuan, respectively. In the past six years, Bilibili has accumulated a loss of more than 7 billion yuan. However, this is mainly because of the resulting higher sales and marketing costs. This expenditure has surged from 1.2 billion yuan in 2019 to 3.49 billion yuan in 2020, and its proportion in total revenue has also increased from $17.7 \%$ to $29.1 \%$. Bilibili also relies heavily on the content provided by video creators and anchors. Professional User Generated Video (PUGV) accounted for $91.4 \%$ of the total Video visits in 2020, compared with $90.1 \%$ in 2019. As the business develops, Bilibili's remuneration will increase accordingly. Although Bilibili got profit through games in recent two years, which effectively expanded the source of income, it still can't cover the whole marketing and contents of the high cost. Nevertheless, the number of users is increasing sharply. To be more specific, in the fourth quarter of 2020, Bilibili monthly active users grew by $55 \%$ year on year, reached 202 million. In 2020, the average monthly active users of Bilibili's mobile terminals has reached 186 million, up $58.1 \%$ year on year.

There are some similarities or differences in getting profit. First, in terms of revenue structure, iQIYI's revenue mainly comes from membership services, advertising, content distribution, and other revenue. In 2020, iQIYI's membership revenue will reach 16.5 billion yuan, which showed an increase trend compared with the pass, up $14 \%$ from 2019 . This raise was mainly due to the increase in subscription membership due to the outbreak of COVID -19 in the first half of 2020, as well as exclusive content releases and various operational plans for the year. Compared with 6.8 billion yuan in online advertising, the revenues are 2.7 billion yuan in content distribution, and 3.7 billion yuan in other revenue. With regard to Netflix, $98 \%$ of the revenue comes from the subscription's membership fees.

Generally, it seems that Bilibili's operation is better than iQIYI and Netflix as the company has more methods to get a stable profit. On this occasion, Bilibili will perhaps take the most significant status in China or even substitutes YouTube in the future.

\section{PUBLICITY - NEW YEAR' S EVE}

When Bilibili was launched in 2009, it was positioned as a quadratic element site. Subsequently, 2014 was positioned as a two-dimensional vertical field. It has launched an animation contract program, which is officially moving into copyrighted video. Since then, Bilibili has targeted young people as its main audience. It was authorized to broadcast by Tokyo Anime Network in 2016, which has been in the leading position in the field of animation video in China. Whereas, Bilibili faces a serious issue after 2016: how to expand its business and consolidate its user base.

Bilibili is trying to expand the content of the plate, attract multi-level age groups, and further sign investment agreements with various enterprises to expand the scope of business. Through a series of event marketing and function expansion, Bilibili is gradually getting rid of the bondage of a single niche platform and transforming into a multi-dimensional, interactive, symbiotic and highly communicable social media [13]. In 2019, the New Year's Eve party with the theme of "The Most Beautiful Night of 2019" was jointly held with domestic authoritative media XinhuaNet, which played an important role in its transformation. The event was also viewed 80 million times online, and Bilibili's market value soared as well, jumping 12.51 percent to 
$\$ 6.54$ billion in trading on the first day after the festival, up 5 billion yuan from the previous trading day.

This is the embodiment of successful transformation, and the background of successful transformation is the success of the Bilibili party form, the integration of different Chinese and foreign cultures. All kinds of culture are presented through packaging and re-creation to conform to the expression habit of youth's mainstream culture and cognition of The Times (e.g., Youth subculture and mainstream culture, mass culture and high culture, traditional culture and popular culture, Chinese culture and western culture) [14].

In essence, it improves the recognition and reputation of the platform, creates high-quality content, enhances the reputation of the media and the media platform, promotes content upgrading, establishes sustainable user relations, and improves user engagement. In terms of commercial value, it is a symbol of enterprise integration and reuse of resources. The most important of these is to understand the company's current position and the value of sustainability. In this area, the 2019 New Year's Eve Gala is a typical case of Bilibili's successful transformation and positioning. However, when Bilibili jointly held the "2020 Most Beautiful Night" New Year's Eve Party with CCTV in 2020, the US stock market fell by $9.15 \%$ on the same day, and a wellknown forum only rated it 6.5 points overnight, which wiped out 1.656 billion yuan (about 10.9 billion yuan) in market value. The problem behind this is divided reviews from divided users.

The high average monthly active users (202 million) that Bilibili has just reached shows itself is no longer a niche company. On account of this, Bilibili has to find a diversified way to operate to meet the needs of the majority of its users. This was best illustrated at the party on the last day of 2020. In terms of program arrangement and form, Bilibili is closer to the standard of major satellite TV platforms. Whereas, this just loses the uniqueness of Bilibili itself, which also leads to a sharp decline in the popularity rating of the gala [15].

Bilibili must have faced a dilemma when trying to craft the 2019 gala, as Bilibili needed to balance the needs of its user base. The main question is about to cater to the masses to get a larger user base or to maintain their style and filter users.

The choice for Bilibili's performance at the 2020 gala is in favor of the former. Obviously, there are more and more homogeneous shows at present. If we examine Bilibili shows according to the standard of satellite TV, we will find that Bilibili shows are not mature and lack of competitiveness. Besides, the show was not quite as impressive as last year's for all its uniqueness. The fundamental reason also lies in that Bilibili did not make a decisive decision, but hesitated about the direction of its transformation. Therefore, it was stuck in an awkward position. Even if the 2020 party doesn't go well, Bilibili is still attracting a lot of users from a financial perspective [16]. For the existing users, Bilibili does not need to cater to them deliberately. Since Bilibili has a strong diversity in the content section, it can push following the needs of users, which has been able to obtain the dependence of users to a great extent. What Bilibili should be thinking about now is how to achieve a positive reputation at the New Year's Eve party in 2021. Assuming that the New Year's Eve party in 2021 follows the pattern of other satellite TV platforms, we can expect its reputation and market value to take another hit. Bilibili should also consider this issue internally, i.e., ACG-related content will likely account for the majority in this year's New Year's Eve party. Meanwhile, in order to attract users from other circles, it is most likely to adopt a way of content combination, combining trendy things with ACG elements to perform in the form of songs and dances. For events with high social attention, they may be presented in the form of second creations [17].

We can also predict that this year's New Year's Eve party will be based on user portraits and invite celebrities with high user favorability. At the same time, it may avoid the problems of last year's gala, reduce the amount of advertising investment, and present the success of the 2021 New Year's Eve party mainly with content. In this case, it is of great significance to Bilibili and will also become a turning point of its transformation.

\section{OPERATION AND EFFICIENCY}

\subsection{Revenue}

According to the financial information released by the BILI.US, the operating revenue of this company mainly consists of 4 parts (Mobile games, Value-added services, Advertising, E-commerce, and others). Comparing the weights of these four sectors in the annual operating revenue, a clear feature can be seen that most of the revenue was gained from Mobile games in the early stage of Bilibili. Despite that mobile games are bringing Bilibili a massive amount of profit and the games themselves are highly matured realizable business mode, it is still harmful to a public limited company to rely heavily on one single profit source and makes the revenue structure of the company unstable. The company has been considered as a game company packaged as a video website for a long period. Bilibili has over 30 mobile games and most of the gameobtained revenue comes from the three specific games: Fate/Grand Order, Azur Lane, Arknights. 


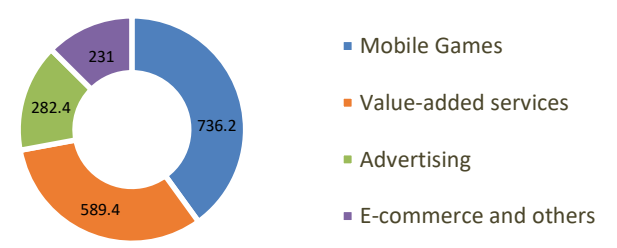

Figure 1 Year 2020 revenue distribution of Bilibili

Not surprisingly, the company has fully aware of their serious situation. Besides, we can see from the data that the proportion of mobile games revenue keeps falling every year. Relatively, the percentage accounts for the revenue gained from the other three sources increased significantly. In 2020, revenues from VAS were US $\$ 103.7$ million in 2020 , which represents an increase of $134 \%$ from 2019. Similarly, revenues from advertising increased to US\$282.4 million and revenues from e-commerce and others increased to US\$113.5 million, representing an increase of $126 \%$ and $109 \%$ from 2019, respectively.

Although mobile games are becoming less important to the firm, they are still the only main way to profitability. In the Fiscal Year 2020, Bilibili earned US\$736.2 million from mobile games and it is around $40.9 \%$ of the total net revenues (US $\$ 1.8$ billion). However, to show the great efforts that the company has done to improve the balance and diversification of its revenue streams, the proportion of the mobile game's revenue to the overall revenue is $61.7 \%$ (US $\$ 103.7$ million) in 2018 and $53.1 \%$ (US\$103.7 million) in 2019. This figure is expected to drop continuously as a result of the rapid development of the company's other businesses.

\subsection{Operation}

Not only the more perfect revenue structure supports the company Bilibili to develop fast, but the user stickiness of this special video-sharing platform is also a big key factor to the success of Bilibili. As a company that mainly faced the younger generation, it is recognized as the fourth largest video website giant. Its average monthly active users (MAUs) reached 202.0 million in 2020 and the average daily active users (DAUs) reached 54.0 million. The most exciting thing about the user stickiness is that the average daily time users spent on this website reached 81 mins, which is a lot higher than many other similar websites.

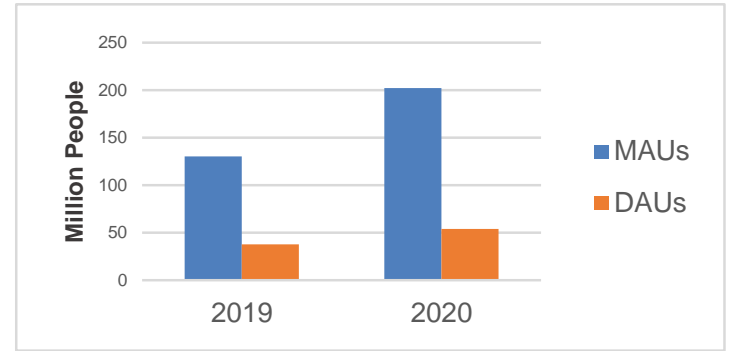

Figure 2 MAUs and DAUs for Bilibili in 2019\&2020

Unlike any other Chinese video website, there is no compulsory viewing of advertisements on Bilibili. Besides, a huge part of the content on this platform is Professional User-Generated Video (PUGV) while most of the other video websites are based on Occupationally-Generated Video (OGV) like Netflix. These amazing characteristics enhance the user stickiness as well as their loyalty to the platform.

The large population of users and their high loyalties make the reforms and developments of Bilibili possible to happen. The growth of VAS is largely attributed to the increasing average monthly paying users (MPUs), which reached 17.9 million in 2020. This is a $103 \%$ increase from the same period in 2019, which can be ascribed to the large amounts of anime copyrights owned by Bilibili and a huge part of its users are anime fans. By charging fees for watching the animations (premium membership program etc.), the firm can increase the VAS revenue in a fast and easy way.

\subsection{Financial Situation}

Since the company went public in 2018 , its stock price per share has already risen more than 15 times from its original opening quotation (US $\$ 9.8$ per share) and it can be seen as a huge success that Bilibili has reached today's height. However, if we research deeper into its financial information, it is obvious to find out that this company is losing money every year.

In the year 2018, the net loss is US\$82.1 million. This number climbed rapidly and reached US\$187.2 million in 2019 and US\$468.1 million in 2020. The word "Loss" is usually be considered as a pejorative term, but in finance, it is a neutral term instead. Many famous companies (e.g., Amazon and JD) all experienced a loss during their periods of rapid development. Similarly, to attract more users and increase their user stickiness as well as loyalty in a higher level, Bilibili has to sacrifice some of their profits and even bear a loss to fight for a larger company scale and bigger market share.

As we have mentioned before, Bilibili is a videosharing platform with no compulsory viewing of advertisements, which also has a lot of exclusive copyrights of animations. These all cost the company quite a lot of money. However, none of these expenses 
is the cause of the loss. The main reason for the company to lose money every year is their growing operating expenses which include Sales and marketing expenses, General and administrative expenses, and Research and development expenses. The total operations expenses have grown from US\$230.4 million in 2018 to US\$916.7 million in 2020 and among the three general operation-expense sectors. Thereinto the money spent on sales and marketing increases the most (US\$450 million).

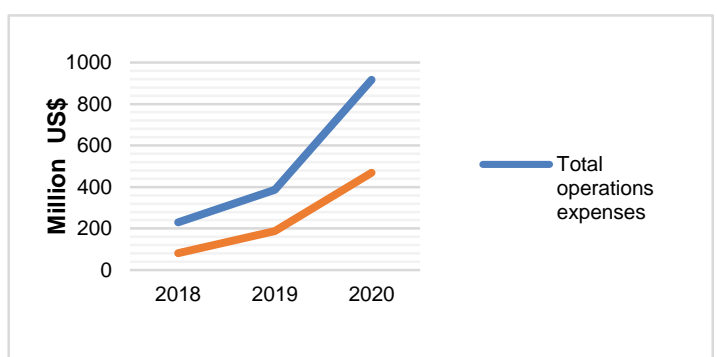

Figure 3 Total operations expenses \& Net loss for Bilibili from 2018 to 2020 ( US \$ In thousands )

Nevertheless, shareholders of BILI.US paid for the loss in order to help company survive. This firm gained financing every year since it went public and this money can back up the temporary loss of Bilibili in return for higher profits earned in the future. The gross profit is positive and growing each year, i.e., it has the increasing ability to gain profits. The positive number of cash in account also indicates that this company is running healthily as well as able to handle financial emergency incidents.

With all these nice-looking indicators, the market and most of the shareholders have confidence and hope for the future of the company, which, clearly shows on the stock price of Bilibili. Although this company has already on the right track, some suggestions can still be proposed base on the current situation of the firm itself.

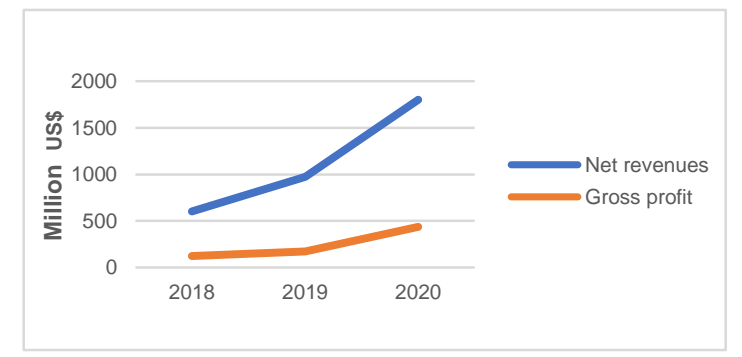

Figure 4 Net revenue \& Gross Profit for Bilibili from 2018 to 2020 (US \$ In thousands)

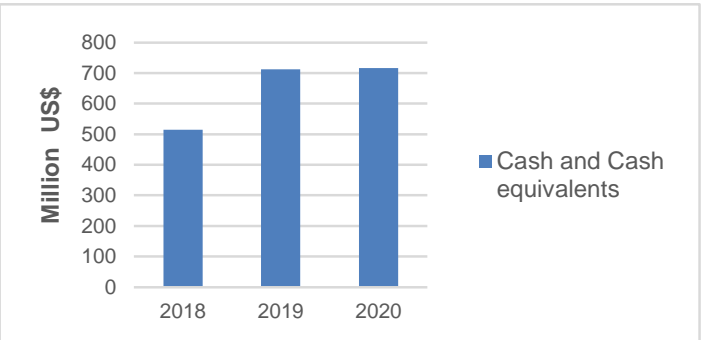

Figure 5 Cash and Cash equivalents for Bilibili from 2018 to 2020 (US \$ In thousands)

\subsection{Suggestions}

As a matter of fact, there are still some other issues that can be fixed to enhance the strength of the company. Although it is generally considered that a more diversified platform's environment caused by the rapid growth of the new user's population is a good thing in a large picture, some side effects do bring a few non-negligible drawbacks. Primarily, the multicultural environment may lead to the antipathy of those original users who were purely attracted to the website by the old unique ACG culture. Instead of keep expanding the number of users, Bilibili should focus more on the using feelings of their most loyal group of users who willing to spend money on the website without hesitation. Finding the solutions to satisfy both the new and old users should be on the main target list of the firm in the future.

Another key problem that the company has to tackle as soon as possible is the increasingly poor quality videos. This is also a side effect caused by the PUGV content. As all the users can upload their creations easily, there is no doubt that the average quality of videos on Bilibili will decrease. The lower quality of contents will lead to the decline of user's stickiness to, which will cause a lower revenue obtained ultimately. A higher standard of video examination might be the way out but it will also raise the barrier of being a video producer on Bilibili and lose part of its users. Therefore, the company still has a long way to go to become the industry benchmark.

\section{VALUATION}

\subsection{DCF}

First, calculate the Weighted Average Cost of Capital (WACC) as the discount rate. Because Bilibili was listed in NASDAQ in 2018, the U.S. five-year Treasury bond interest rate is used as the risk-free interest rate Rf. According to the historical statistics, the U.S. five-year Treasury bond interest rate was $0.8164 \%$ on 25 th April 2021, i.e., let $\mathrm{Rf}=0.8164 \%$. $\beta$ is the risk coefficient of the company and the adjusted beta coefficient is used in this article because it reduces the influence brings by the fierce variation of the market. 
According to Bilibili Inc.'s annual report and S\&P 500 adjusted beta coefficient $=$ original beta coefficient $* 0.67+0.33=1.4$. Market Profitability Rm adapts S\&P 500 's profitability rate which is equal to 4180.17 on 25th April 2021. 93 years ago, on 25th April 1928, the $\mathrm{Rm}=17$.

As a result, the geometric mean of the interest rate is:

$$
\mathrm{Rm}=\sqrt[93]{\frac{4080.17}{17.26}}-1=6.05 \%(5.1)
$$

i.e., Financing cost is:

$\mathrm{Ks}=\mathrm{Rf}+(\mathrm{Rm}-\mathrm{Rf}) \times \beta, \mathrm{Ks}=0.82+(6.050 .82) \times 1.4=8.142 \%$

In recent years, Bilibili's long-term liability of longterm debt and short-term liabilities are all equals to 0 , this essay equals WACC to $\mathrm{Ks}=8.142 \%$. Free Cash Flow is a measure of financial performance calculated as Net Cash Flow from Operations minus Capital Expenditure. Bilibili Inc's Free Cash Flow is part of a valuation analysis module that helps investors searching for stocks that are currently trading at higher or lower prices than their real value. The 10 years trend of Bilibili's FCF is increasing slightly volatile. Since FCF = Cash from Operations - CapEx, the next following years' FCF can be estimated on the table. Bilibili Inc Market Capitalization is most likely to increase significantly in the upcoming years. The last year's value of Market Capitalization was reported at 30.11 Billion. The current Working Capital is estimated to increase to about 9B, while Capital Expenditure is projected to decrease to $(618 \mathrm{M})$.

Table 1. Free cash flow of Bilibili Inc

(Million Dollars)

\begin{tabular}{cccccc}
\hline Time period & 2016 & 2017 & 2018 & 2019 & 2020 \\
\hline FCF & 14.67 & 17.72 & 39.12 & 54.83 & 78.49 \\
& & & & & \\
Operating & 59.81 & 116.02 & 181.47 & 237.66 & 385.74 \\
cash flow & & & & & \\
& & & & & \\
Capital & 45.14 & 98.30 & 142.35 & 182.83 & 307.25 \\
expenditure & & & & & \\
\hline
\end{tabular}

Note. Adapted from Annual report (2020) by United States Securities And Exchange Commission,2020.
Table 2. Discounted cash flow of Bilibili Inc (Million Dollars)

\begin{tabular}{cccccc}
\hline $\begin{array}{c}\text { Time } \\
\text { period }\end{array}$ & 2019 & 2020 & 2021 & 2022 & 2023 \\
\hline FCF & 54.83 & 78.50 & 109.01 & 148.35 & 199.07 \\
& & & & & \\
& & & & & \\
Discount & 8.1 & 8.1 & 8.1 & 8.1 & 8.1 \\
rate (\%) & & & & & \\
& & & & & \\
& & & & & \\
DCF & 50.72 & 67.17 & 86.30 & 108.65 & 134.85 \\
\hline
\end{tabular}

The data above can be used to estimate the FCF in the following years. Combining the cost of equity, the cost of bonds, and the debt ratio, we can calculate the average cost of capital over the next 5 years. We predict that Bilibili's cost of capital will reach a stable level of around $7 \%$ after 5 years. The formula to valuate a company with DCF model is that:

$$
\mathrm{V}_{0}=\sum_{\mathrm{t}=1}^{\mathrm{n}} \frac{\mathrm{FCFF}_{\mathrm{t}}}{(1+\mathrm{WACC})^{\mathrm{t}}}+\frac{\mathrm{FCFF}_{\mathrm{n}+1}}{(1+\mathrm{WACC})^{\mathrm{n}}} \times \frac{1}{\mathrm{WACC}_{\mathrm{n}}-\mathrm{g}_{\mathrm{n}}}
$$

where $\mathrm{V}_{0}$ is the value of the company and FCFFt is the expected Free Cash Flow in year t. According to the formula it is estimated that the value of the company is about 40 billion dollars.

\section{2. $P / S$}

The price-to-sales $(\mathrm{P} / \mathrm{S})$ ratio is a valuation ratio that compares a company's stock price to its revenues. It is an indicator of the value that financial markets have placed on each dollar of a company's sales or revenues. Compared to other methods, $\mathrm{P} / \mathrm{S}$ is suitable to valuate companies with rapid business expansion but weak profitability in a certain period of time. Bilibili has a perennial loss but its total revenue is increasing dramatically, so it is suitable to adapt the $\mathrm{P} / \mathrm{S}$ ratio.

$$
\mathrm{P} / \mathrm{S} \text { Ratio }=\frac{\mathrm{MVS}}{\mathrm{SPS}}(5.3)
$$

where MVS presents Market Value per Share, SPS presents Sales per Share. Bilibili Inc Sales per Share is increasing over the last several years with slightly volatile swings. Sales per Share is predicted to flatten to 4.51. During the period from 2010 to 2021 Bilibili Inc ADR Sales per Share regressed distribution of quarterly values had a coefficient of variation of 102.56 and $r$ value of 0.83 .

According to the newest data, MVS $=116.66$, and the latest $\mathrm{P} / \mathrm{S}$ ratio on 26th April 2021 is 23.45 . Generally, a company with higher debt will have a lower $\mathrm{P} / \mathrm{S}$ ratio. 
The reason is that some of those sales have to go toward debt interest and paying down debt.Company's market capitalization first multiply the number of shares outstanding by their current market price is the $\mathrm{P} / \mathrm{S}$ ratio. A low $\mathrm{P} / \mathrm{S}$ ratio in comparison to peers could suggest some undervaluation While a high $\mathrm{P} / \mathrm{S}$ ratio would suggest overvaluation. Bilibili has a relatively reasonable $\mathrm{P} / \mathrm{S}$ ratio.

Table 3. $\mathrm{P} / \mathrm{S}$ ratio for Bilibili Inc

\begin{tabular}{ccccc}
\hline Time period & 2018 & 2019 & 2020 & 2021 \\
\hline $\begin{array}{c}\text { Sales per share } \\
\text { Stock price }\end{array}$ & 2.56 & 3.00 & 5.31 & 4.51 \\
( dollars) & 9.96 & 18.62 & 85.72 & 118.46 \\
& & & & \\
P/S ratio & 3.89 & 6.19 & 16.14 & 26.27 \\
\hline
\end{tabular}

Note. Adapted from Annual report (2020) by United States Securities And Exchange Commission,2020.

BILI was compared to 400 industry peers in the Computer Programming, Data Processing, and Other Computer Related Services industry. The financial health of BILI is medium and its profitability is quite bad. BILI has a Return On Assets of $-12.60 \%$, which is below the industry average of $-2.17 \%$. BILI's Profit Margin of $-25.06 \%$ is worse than the rest of the industry. The industry average Profit Margin is $-5.40 \%$ and the Price/Earnings Ratio is negative for BILI. In the last year, negative earnings were reported. Besides, next year BILI is expected to report negative earnings again, which makes the Forward Price/Earnings Ratio negative, too. Although, BILI shows excellent growth, which is consistent with its market value. The Earnings Per Share is expected to grow by $55.73 \%$ on average over the next 2 years and this is a very strong growth. BILI shows strong growth in Revenue, which has been growing by $69.24 \%$ yearly over the last 5 years.

\section{CONCLUSION}

In summary, we review the development of Bilibili and carry out value analysis of the company from four parts. Primarily, the competition is conducted among Bilibili, iQIYI and Netflix. The next is Business bottleneck and transformation. Subsequently operation mode/profit direction has been discussed. Eventually, the valuation of Bilibili is analyzed with the method of $\mathrm{DCF}$ and P/S ratio. Generally, the situation is that Bilibili has a better prospect than iQIYI and Netflix for its profit model. Besides, Bilibili is a representative case of effective transformation and positioning because of a New Year's Eve party. By the analysis of revenue and operation, although this company is on the right track, there are still some suggestions to let Bilibili more stable in operating. Moreover, it is estimated that the value of the company is about 40 billion dollars and shows a significant growth in total revenue. In the prospective view, Bilibili is an outstanding company born in the video track, which has successfully gained a large number of users and fans in a short time. Through research and investigation, this article finds that the company has already reached the expected valuation, and may have a huge potential in the future.

\section{REFERENCES}

[1] Hong Liu, \&Qian Wang. (2020). Circles Communication: The Underlayer Logic of a Media Productization Taking the New Year's Eve Party of Station B as an Example.Young reporter.

[2] Fu Xiaoguang, \& Lin Xinke. (2020). Analysis of the Popularization Path of Circle Culture - Analysis of Bilibili2019 New Year's Eve Party.Journalism and Writing, 2.

[3] Gao, Y. (2020). Analysis on the marketing success factors and deficiencies of the New Year's Eve Gala at Station B.To write.

[4] Yifei Zhao \& Yicheng Zhao (2020). Optimization of the Financial Strategy of Bilibili Barrage Website from the Perspective of Social Responsibility. In Journal of Finance Research(pp. 45-50).

[5] Xiaowen, Yuan(2020). Quality analysis of cash flow of Bilibili Inc. In Inner Mongolia Science Technology Economy(pp.54-57).

[6] Fei Gao, Ting Ni, \& Yulin Song (2019). Sustainability of Bilibili Enterprise Profit Model Based on Financial PerspectiveIn Fortune Today, 000(005), (pp.121-122).

[7] Bingyu Jiang. Research on Bilibili Profit Model Based On Financial Analysis. Northeast Petroleum Univeristy.

[8] Meng Zhu. (2018). Business model analysis of Bilibili barrage website. Development and reform,theory and practice of No. 17 (05), 30-33 + 41.

[9] Jingdan Weng \& Shengyun Dai. (2018). Comparison between Letv and Bilibili operation mode. Taxation,000(015), P.255-255.

[10] Ke Rong, Fei Xiao, Xiaoyu Zhang, Jingjing Wang (2019). Plat- form strategies and user stickiness in the online video industry. In Technological Forecasting Social Change 143 (2019) 249-259. 
[11] Hsiu-Sen Chiang Kuo-Lun Hsiao, (2015), "YouTube stickiness: the needs, personal, and environmental perspective", Internet Research,Vol. 25 Iss 1 pp. 85 - 106

[12] Keng-Chieh Yang, Chia-Hui Huang, Conna Yang, Su Yu Yang, (2017) "Consumer attitudes toward online video advertisement:YouTube as a platform", Kybernetes, Vol. 46 Issue: 5, pp.840-853

[13] Yao, X., \& Ji, Z. (2020). Research on the Transformation Path of Bilibili Danmu.com to Social Media. New media research.

[14] Hong Liu, \& Qian Wang. (2020). Circles Communication: A Low- level Logic of Media Productization - Taking the New Year's Eve Party of Station B as an Example.Young reporter.

[15] Renfei Huang. (2016). Discussion on the Transformation and Change of Otaku Culture in the New Media Era: A Case Study of Bilibili Bombard.com. Journalism and Communication, 6,16-18.

[16] Zhixiao Guo (2014). The Value Evaluation of TED Baker Company Based on DCF Model. In Enterprise Management. (pp. 44-45)

[17] Min Wan (2010). Method and application of overall valuation of companies Cooperative $\mathrm{E}$ Economy Science. (pp. 44-46) 